\section{The Time Has Come!}

$\mathbf{W}_{\mathrm{red}}^{\mathrm{h}}$ hen imatinib was approved by the FDA almost 20 years ago, a passionate prediction arose that, going forward, all targeted therapeutics would be approved without regard for the primary site of disease, based solely on the presumed molecular driver. I said, "No! That won't work." A driver for one cancer may not be a driver for another. There is also the fact that we have successful chemotherapy treatments for many cancers-who would want to mess with that paradigm?

Well, of course, I was wrong. And the FDA has been very receptive to the concept of crosscutting approvals. Thank you!

With the breakthrough of immuno-oncology, we now have pembrolizumab approved for any patient with a microsatellite instability (MSI)-high or mismatch repair (MMR)-deficient tumor. And now, larotrectinib, an inhibitor of tropomyosin receptor kinase that is highly active against an NTRK gene fusion, has been approved across all disease types.

OK, then, wake up! How do we find these patients? The new therapies mean we must perform MSI/MMR testing and molecular profiling of tumor tissue for everyone, at least those with metastatic disease. How else would we find patients eligible for therapy with these new treatments?

Then what are the barriers? The first is the adequacy of the diagnostic biopsy sample, which is likely to be accessed for analysis. To begin, we need to make sure our diagnostic biopsies contain enough material for ancillary molecular analyses. This isn't trivial. This will generally require more than the usual fine-needle aspiration squirt of sample that can be used for diagnosis. Increasingly, we are gaining confidence in analyses derived from cell-free DNA in blood or other body fluids. So we should be able to sort this out.

The next barrier is cost. However, the beauty of all this is that because of advancing technologies, the cost of these analyses is decreasing and the market competition is robust. We are not testing for single abnormalities but instead for a broad panel of aberrations that could be clinically actionable. Insurance providers will be faced increasingly with this request. Fortunately, NCCN Guidelines are incorporating the need for these studies. This mandates reimbursement at least from the Centers for Medicare \& Medicaid Services, and we hope private insurers will follow suit.

The other niggling issue here is that, without a doubt, we will find molecular abnormalities in tumors that reflect germline mutations. This obviously leads to the need for genetic counseling and germline testing for mutations. But I consider that a bonus. We can help these families. We just need to be prepared to deal with the increased need for counseling and screening.

Without a doubt, the era of precision oncology is here, and I expect many more breakthroughs will come soon. Just as we now order a "chem" panel when we may only need to check bilirubin, we will now order broad molecular diagnostics on a variety of specimens.

The time is here. The time is now. Are you ready? Let's go! JNCCN@nccn.org or log into www.editorialmanager.com/JNCCN.

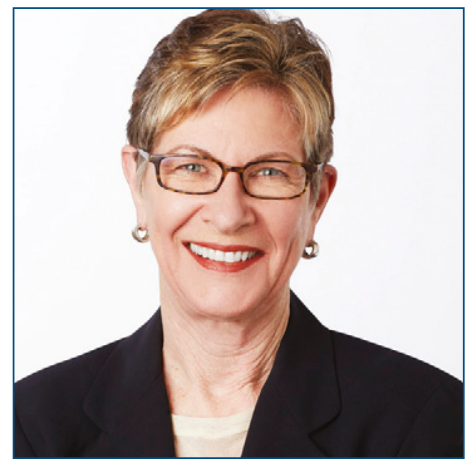

MARGARET TEMPERO, MD

Margaret Tempero, MD, is a Professor of Medicine and Director of the UCSF Pancreas Center and editor-in-chief of JNCCN. Her research career has focused on pancreatic ductal adenocarcinoma, especially in the area of investigational therapeutics. Dr. Tempero has served on the ASCO Board of Directors and as ASCO President. She currently serves on the ASCO Conquer Cancer Foundation Board. She codirected the AACR/ASCO Methods in Clinical Cancer Research and taught this course and similar courses in Europe and Australia. She was founding Chair of the $\mathrm{NCl}$ Clinical Oncology Study Section and served as a member and Chair of the $\mathrm{NCl}$ Board of Scientific Counselors Subcommittee A. She is a member of the Scientific Steering Committee and Chair of the Clinical and Translational Study Section for the Cancer Prevention \& Research Institute of Texas. She is or has been on the Scientific Advisory Boards of the Lustgarten Foundation, the Pancreatic Cancer Action Network, the $\checkmark$ Foundation, The Alberta Canada Cancer Board, and the EORTC. She served as a member of the Oncology Drug Advisory Committee for the FDA. She has served as Deputy Director and Interim Director for the UNMC Eppley Cancer Center. She is Chief Emeritus of the Division of Medical Oncology at UCSF and served as the founding Deputy Director and Director of Research Programs at the UCSF Helen Diller Family Comprehensive Cancer Center.

doi: $10.6004 /$ jnccn.2019.0020

The ideas and viewpoints expressed in this editorial are those of the author and do not necessarily represent any policy, position, or program of NCCN. 